\title{
High power electron diode for linear induction accelerator at a flash radiographic facility
}

\author{
J. M. Plewa, ${ }^{1}$ V. Bernigaud, ${ }^{2}$ T. Barnes, ${ }^{2}$ F. Poulet,${ }^{2}$ A. Georges, ${ }^{2}$ R. Delaunay, ${ }^{1}$ M. Ribière, ${ }^{1}$ \\ C. Vermare,${ }^{3}$ M. Yousfi, ${ }^{4}$ O. Eichwald,${ }^{4}$ T. d'Almeida, ${ }^{1}$ and R. Maisonny ${ }^{1, *}$ \\ ${ }^{1}$ CEA, DAM, GRAMAT, F-46500 Gramat, France \\ ${ }^{2} C E A, D A M, V A L D U C, F-21580$ Salives, France \\ ${ }^{3}$ CEA, DAM, CESTA, F-33114 Le Barp, France \\ ${ }^{4}$ LAPLACE, CNRS, University of Toulouse, 118 Route de Narbonne, F-31000 Toulouse, France
}

(Received 28 March 2018; published 31 July 2018)

\begin{abstract}
We investigated a new cathode design and beam transport with the EPURE axis-1 injector in order to increase the beam characteristics at $3.8 \mathrm{MeV}, 80 \mathrm{~ns}$ FWHM from the $2.0 \mathrm{kA}$ nominal current to $2.6 \mathrm{kA}$ corresponding to an average current density of $82 \mathrm{~A} / \mathrm{cm}^{2}$. Such current increase is highly desirable for improving the $\mathrm{x}$-ray dose and hence radiographic performances. To achieve this, a time-dependent model based on the particle-in-cell method was developed in order to simulate the injector. Using results from calculations based on this model, a $17.2 \mathrm{~cm}$ AK gap diode with a larger radius cathode $(3.175 \mathrm{~cm})$ was designed, manufactured and tested. Experimental and calculated currents and emittances are qualitatively compared. The study provides a detailed understanding of the beam dynamics inside this type of high current, high energy injector.
\end{abstract}

DOI: 10.1103/PhysRevAccelBeams.21.070401

\section{INTRODUCTION}

$\mathrm{X}$-ray flash radiography is a powerful diagnostic used worldwide [1-6] for investigating the structural response of matter under impulsive loading during hydrodynamic experiments. Intense pulsed $\mathrm{x}$-ray sources are required for capturing target motion during these experiments and, hence, obtaining good quality images. A joint U.K./ France hydrodynamics research facility known as EPURE is under construction at CEA VALDUC in France. At the present stage, this facility operates in a single radiographic axis configuration. This first axis generates an intense $\mathrm{x}$-ray pulse resulting from the interaction of a $2 \mathrm{kA}, 19 \mathrm{MeV}$, 80 ns FWHM electron beam [7] with a solid tantalum target. The resulting bremsstrahlung spectrum is required to penetrate high Z, highly compressed objects during hydrodynamic experiments. By 2022, two additional high performance radiographic axes will be added and this unique triple-axis radiographic facility will be operated jointly by British and French teams. One of these two upcoming x-ray sources will be a linear induction accelerator (LIA) similar to the current first axis accelerator.

\footnotetext{
"remi.maisonny@cea.fr
}

Published by the American Physical Society under the terms of the Creative Commons Attribution 4.0 International license. Further distribution of this work must maintain attribution to the author(s) and the published article's title, journal citation, and DOI.
Recently, significant efforts were undertaken in order to enhance the current first axis radiographic performance such as x-ray dose and spot size. This required a thorough numerical modeling and prediction of the beam dynamics within the injector of the linear induction accelerator. The objective of the present study is to provide such a comprehensive numerical analysis based on particle-in-cell (PIC) simulations. Section II provides an overview of the first axis technical features. In Sec. III, the experimental setup and the overall geometry used for the numerical simulations are presented. Next, simulation results are discussed and compared to experimental measurements performed at the EPURE facility in order to increase the beam intensity. This was achieved by using an optimized velvet cathode, calculated numerically, to increase intensity from 2.0 to $2.6 \mathrm{kA}$. Finally, the main concluding remarks about this numerical approach are drawn.

\section{BACKGROUND}

The first axis uses 64 ferrite-loaded cells to accelerate the beam produced inside the injector where a high power electron diode, composed of a cathode and an anode, is used to generate an intense high quality, pulsed electron beam. For this purpose, a velvet cathode is driven with a $75 \Omega$ graded transmission line, which delivers a $3.8 \mathrm{MV}$, $95 \mathrm{~ns}$ (FWHM) high power electrical pulse across a $17.2 \mathrm{~cm}$ diode gap. The application of a strong electric field results in plasma formation due to surface flashover $[8,9]$. This electric field extracts a space charge limited 
electron flow from the plasma. Space charge limited (SCL) flow in diodes has been an area of active research since the pioneering work of Child and Langmuir [10,11]. The scope of the theory was later extended in order to incorporate other physical mechanisms such as relativistic behavior [12] or quantum mechanical effects [13], although the modeling was limited to a one-dimensional treatment of the SCL flow. In 2001, Lau first introduced a simple analytical theory for two-dimensional Child Langmuir law [14] which proved to be in good agreement with the simulation data obtained by Luginsland [15]. In spite of these theoretical advances, multidimensional codes remain necessary tools for gaining insight into the physics of multidimensional space charge limited flow that takes place within the injector diode. More recently, Coleman and co-workers [16] carried out a quantitative study of the plasma dynamics within the DARHT-1 high power diode to gain a better understanding of the electron beam generation. Diode transport simulations were performed, in the injector of the FXR induction accelerator [17], in order to lower the beam emittance which is a contributing factor that affects radiographic spot size [18].

\section{SIMULATION MODEL AND EXPERIMENTAL VALIDATION}

\section{A. Numerical configuration}

Particle-in-cell simulations were used to study and predict beam dynamics inside the injector. Simulations were performed with the fully relativistic 3D LSP code [19] used here in $2 \mathrm{D}$ cylindrical geometry $(\mathrm{R}, \mathrm{Z})$. Figure 1 shows the overall simulation geometry.

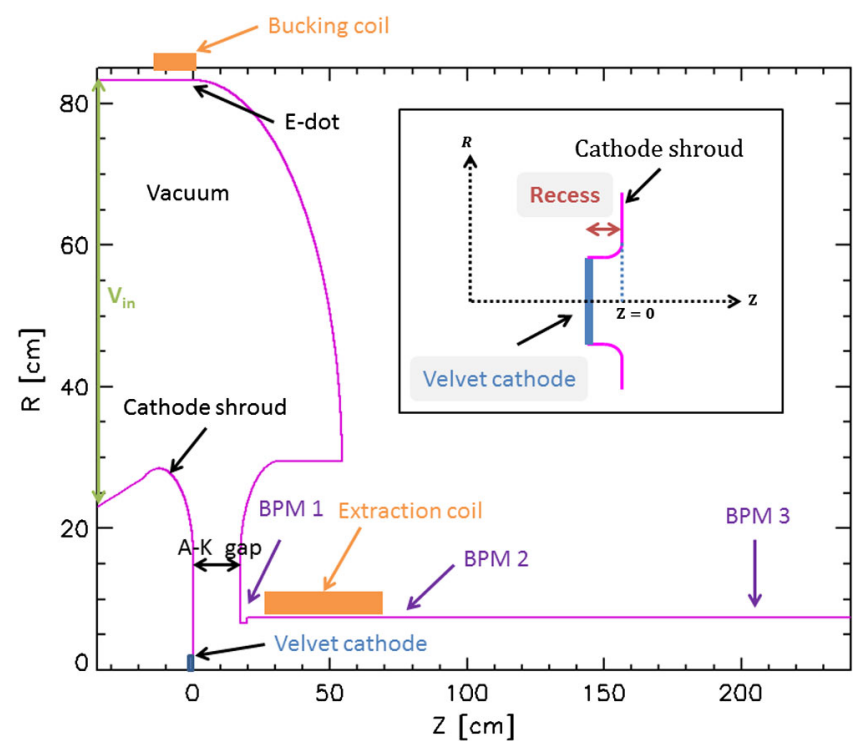

FIG. 1. Geometry used for simulating the beam dynamics inside an injector of an induction accelerator. BPM (for beam positioning monitor) indicates the location of the current sensors. The inset is a zoom in the diode region.
The emission is modeled by a SCL flow at the cathode with an electric field threshold of $20 \mathrm{kV} / \mathrm{cm}$. The latter is a mean value obtained from a dedicated bench test experiment. The $3.5 \mathrm{eV}$ average electron emission temperature used in this study was inferred from spectroscopic measurements made on the EPURE axis-1, based on a collisional-radiative model [20]. All other materials (stainless steel and aluminum) are treated as time-accurate Integrated TIGER Series (ITS) media (Monte Carlo treatment) for simulating electron scattering and energy losses [21]. The diode extraction voltage, $V_{\text {in }}$, used in LSP is the experimental value measured by a flush mounted coaxial E-dot at EPURE (Fig. 2). The amplitude and time profiles of the experimental data are properly incorporated in the simulation. The beam position monitors (BPM 1,2 and 3) indicate the location of the sensors which provide the experimental current. A grid resolution of $250 \mu \mathrm{m}$ was used. The LSP code was run with an explicit particle push and a time bias explicit field solver to damp high frequency noise, the typical time step was $0.5 \mathrm{ps}$.

After being produced within the diode, the beam is extracted and accelerated across 64 ferrite-loaded cells. The value of the extraction magnetic field is critical for matching the beam with the desired envelope parameters in order to avoid emittance growth which may result from envelope oscillations during the transport. The use of a magnetic field for extracting the electron beam at the velvet cathode is likely to affect the radiographic spot size at the end focus. Therefore, a "bucking coil" with reverse polarity is implemented in order to suppress the axial magnetic field at the center of the cathode (at $R=0 \mathrm{~cm}$ ) and, hence, reduce its value at larger radii. Magnetic field measurements were carried out for each solenoid in the accelerator in order to determine the best fit model. Figure 3 shows the

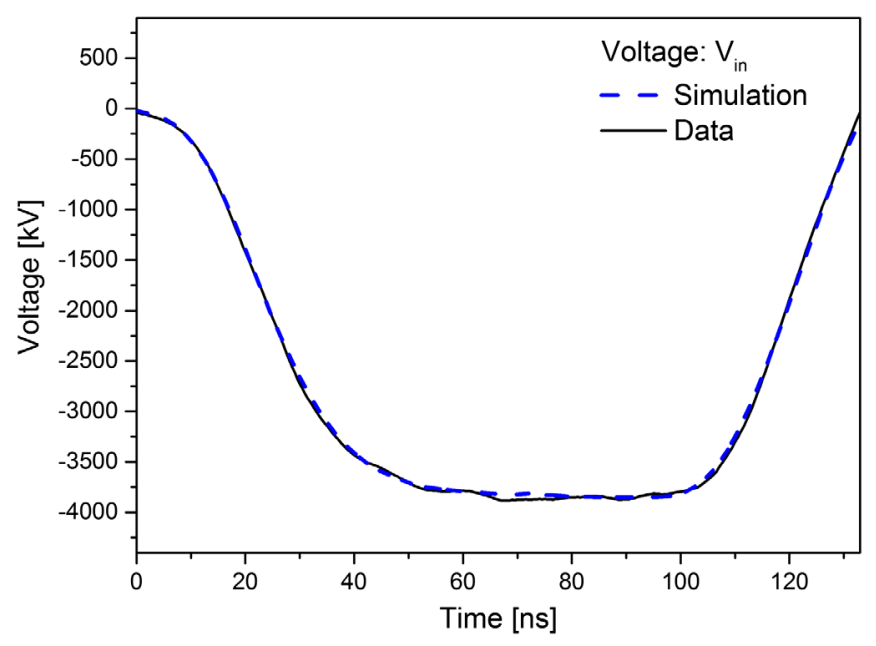

FIG. 2. Typical measured voltage on EPURE axis-1 (black) and simulated voltage (dashed blue) used as an inlet boundary in the simulation ( $V_{\text {in }}$ in Fig. 1). 

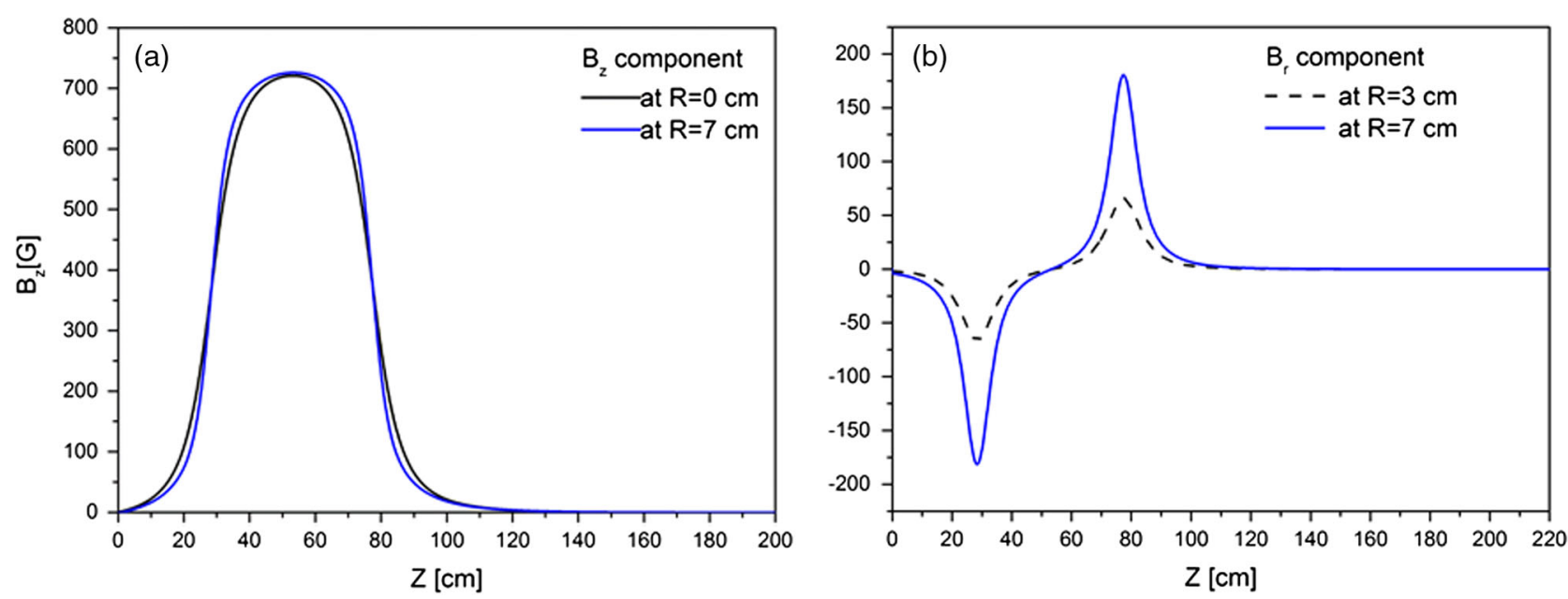

FIG. 3. Simulated magnetic fields used in the simulation for a 201 A driving current on the extraction solenoid when the bucking coil current is chosen to minimize the on axis magnetic field at the velvet cathode; (a) $B_{z}$ at $R=0 \mathrm{~cm}$ (black line) and $R=7 \mathrm{~cm}$ (blue line) as a function of $Z$; (b) $B_{r}$ component at two different radii: at $R=3 \mathrm{~cm}$ (black dashed line) and $R=7 \mathrm{~cm}$ (blue line).

resulting magnetic field as a function of the propagation distance $(\mathrm{Z})$ for various radii used in the simulation corresponding to a driving current of $201 \mathrm{~A}$ on the extraction solenoid.

In the simulation, the off-axis field components $B_{r}$ and $B_{z}$ are inferred from $B_{z}(0, Z)$ and its derivatives [22]. It is important to include all nonlinear components from external fields because they contribute significantly to the beam properties during the transport within both the injector and the linear accelerator $[23,24]$. The specific effects of these nonlinear components on the beam dynamics were accounted for in the PIC calculations.

\section{B. Simulation and experimental results}

We investigated a new cathode design and beam transport through the injector in order to increase the beam

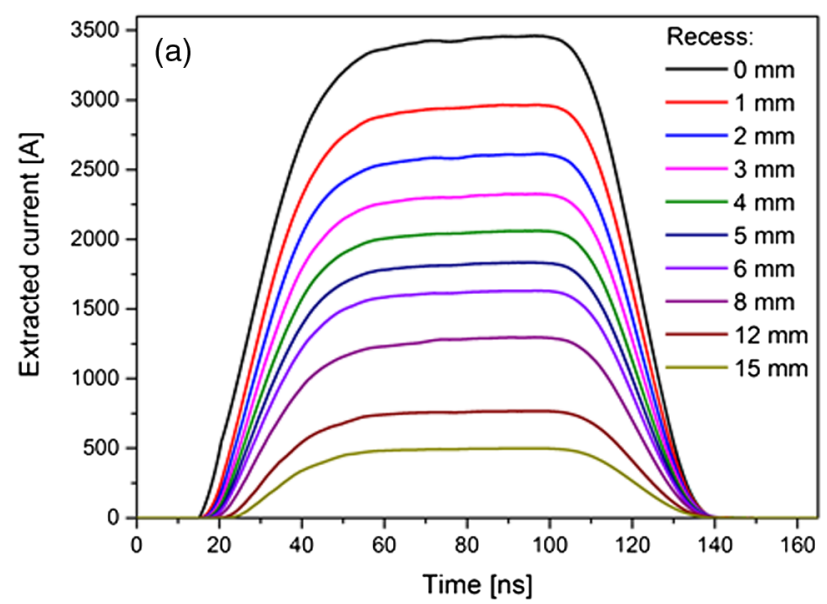

intensity from the nominal 2.0 to $2.6 \mathrm{kA}$. The radius of the velvet cathode was changed from 2.550 to $3.175 \mathrm{~cm}$. Next, results from numerical simulations in this new configuration are discussed and compared to experimental measurements at the EPURE facility.

\section{Diode results}

Usually, the velvet cathode is recessed relative to the shroud, positioned at negative Z, as shown in the inset of Fig. 1. Recessing the cathode has significant effects on the initial beam properties at the cathode. This point is specifically examined in what follows.

The time profile of the simulated anode current at the BPM 1 location is shown in Fig. 4(a) for a velvet cathode with a $3.175 \mathrm{~cm}$ radius, $17.2 \mathrm{~cm}$ AK gap, and recess values ranging from 0 to $15 \mathrm{~mm}$.

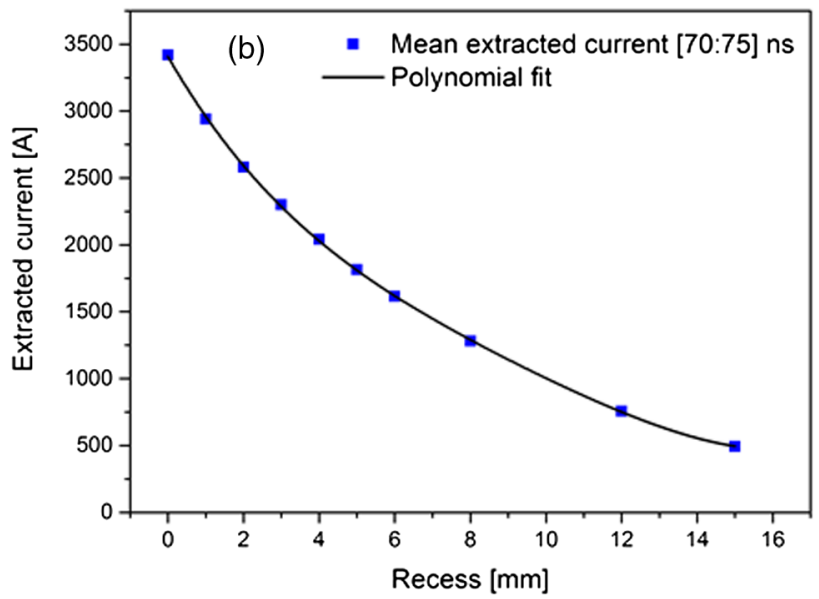

FIG. 4. (a) Simulated electron beam current at BPM 1 as a function of time for different recess values. (b) Simulated mean beam current over [70-75] ns range as a function of recess (blue square) and fourth order polynomial fit (black curve): current $=3408.08-491.40($ recess $)+47.76(\text { recess })^{2}-3.07(\text { recess })^{3}+0.08(\text { recess })^{4}$. 
Figure 4(a) suggests that the extracted current is strongly dependent on the recess value. The mean extracted current over the [70:75] ns time range is plotted as a function of the recess in Fig. 4(b). This evolution is well fitted by the following fourth order polynomial function:

$$
\begin{aligned}
\text { Current }= & 3408.08-491.40(\text { recess })+47.76(\text { recess })^{2} \\
& -3.07(\text { recess })^{3}+0.08(\text { recess })^{4},
\end{aligned}
$$

where the current and recess are expressed in A and $\mathrm{mm}$, respectively. Figures 4(a) and 4(b) show that the beam intensity is strongly reduced when the recess increases. A direct comparison of the effect of a change in cathode radius on the current is relevant only if the recess values associated are identical.

This result is better understood in the light of the results presented in Fig. 5, where simulated electronic charge distribution profiles at the cathode are shown for five recess values.

The features of the beam profiles represented in Fig. 5 are solely governed by the total electric field at the cathode surface. The extracted current is proportional to the total electric field. This total electric field is the addition of the "external" electric field applied to the cathode shroud and the electric field of opposite sign induced by the space charge density. The beam halo profile observed at null recess in Fig. 5 (black curve) is induced by a spatial change in electric field between surrounding vacuum and the beam. At the center of the cathode $(R \leq 2 \mathrm{~cm})$ the total electric field is dominated by the screening effect due to the space charge density. This results in a lower electric field inside the beam than outside. Towards the cathode edges, the electric field screening due to space charge is less pronounced. Therefore, the contribution of space charge

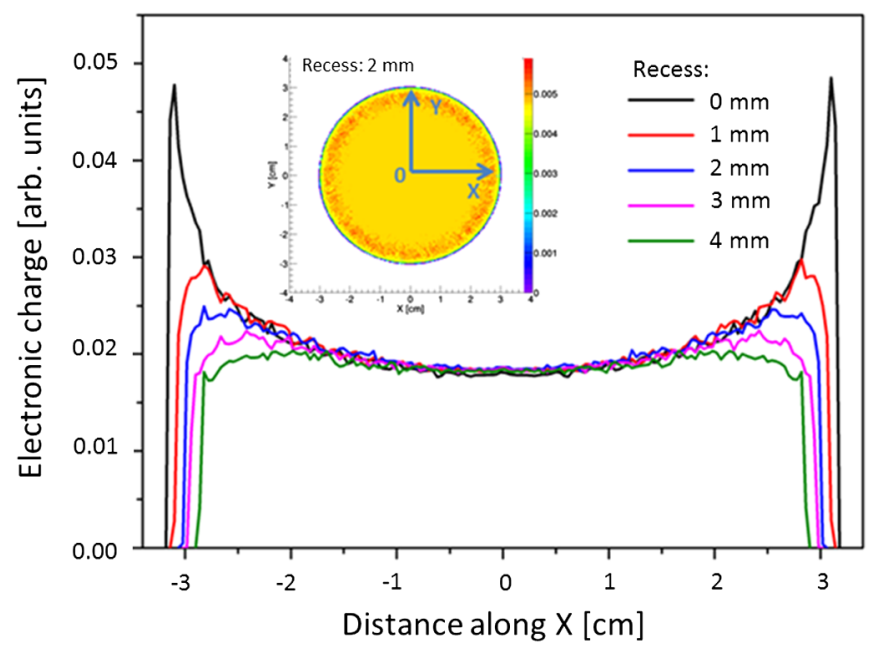

FIG. 5. Simulated electron beam profile at the cathode, along $X$ at $\mathrm{Y}=0$, on the flattop voltage for a $3.175 \mathrm{~cm}$ cathode radius for five recess values ranging from 0 to $4 \mathrm{~mm}$. The inserted image is a 2D plot of the simulated beam distribution at the cathode for a $2 \mathrm{~mm}$ recess. density to the total electric field is less important. Thus, the rise of the overall electric field at the edges results in higher extracted current. In addition, by increasing the recess, the external electric field at the cathode edges is decreased leading to a reduction of the halo beam profile and a reduction of the beam radius, which is consistent with the observed edge profiles in Fig. 5. These results are in good agreement with the study of Hegeler and co-workers [25]. The beam halo profile is penalizing because it is a potential source of emittance growth during beam transport, which is likely to affect the final spot size. Previous experiments performed on DARHT axis- 1 and AIRIX evidenced a change in beam profile occurring while switching from a standard cathode geometry to a Pierce geometry [26].

Based on the time-dependent simulation model developed in this work, a new cathode with a $3.175 \mathrm{~cm}$ radius was designed, manufactured and tested on the EPURE injector. The $2 \mathrm{~mm}$ recess configuration was chosen to reduce the beam hollowness. As can be seen in Fig. 6, a good agreement is obtained between simulated and measured currents at the BPM 2 location (see Fig. 1). It is important to note that the comparison between simulated and experimental currents demonstrated that the emission surface does not coincide with the velvet surface. Considering a 2D uniform emission region, a correct extraction current can only be reproduced if one considers that the emission surface is slightly downstream (of the order of $1 \mathrm{~mm}$ ) of the actual velvet surface. Such an assumption is particularly relevant to the current plateau while it is less valid at times corresponding to the rise and fall of the current profile. This explains the discrepancies between simulated and experimental currents appearing in these regions.

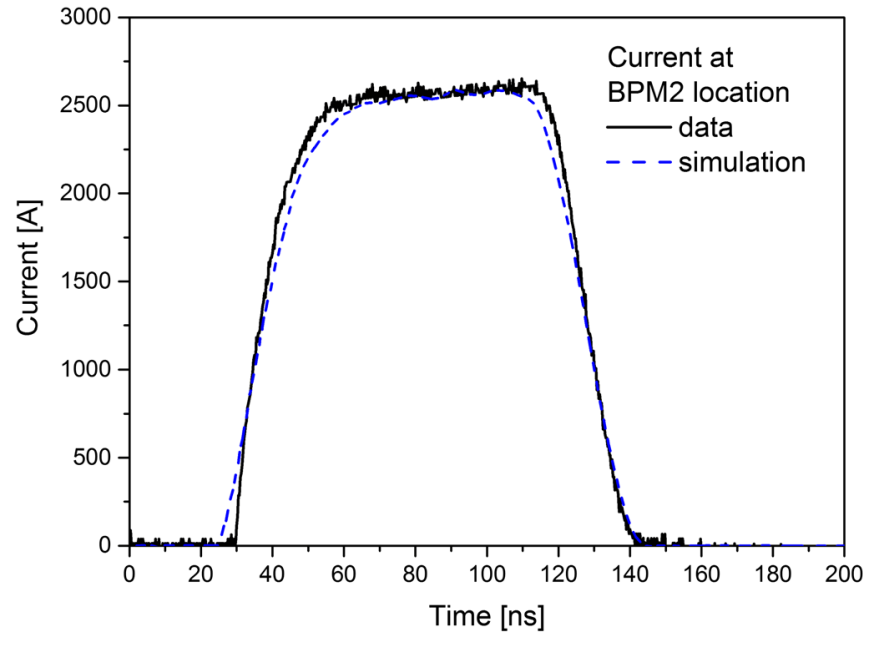

FIG. 6. Experimental and simulated currents at the BPM 2 location for the new $3.175 \mathrm{~cm}$ radius velvet cathode and a $201 \mathrm{~A}$ driving current. 
On the flattop, the experimental current only increased from $2525 \mathrm{~A}$ at $t=60 \mathrm{~ns}$ to $2615 \mathrm{~A}$ at $t=105 \mathrm{~ns}$. Over the pulse duration, the current variation is due to several factors which depend on the diode operation. These factors are the voltage increase, the changes introduced in the diode geometry by the expanding velvet plasma [27] and ion flow from the anode [28]. In our study, the hollowness of the anode and the large AK gap $(17.2 \mathrm{~cm})$ prevents ion flow from the anode during the time window of interest. In order to estimate the plasma expansion velocity it is necessary to build a relationship between current, voltage and plasma expansion velocity. Using our simulation model, a relationship is built between the current and the voltage for each diode geometry, without taking the plasma motion into account. This approach appears to be more accurate than an analytical one because no assumption is made on the relationship between current density and voltage [29].

The good agreement obtained between the experimental and simulated diode justifies to put forward two fourth order polynomial fits of the current as a function of recess at $t=60 \mathrm{~ns}$ and $t=105 \mathrm{~ns}$, respectively. A similar approach was previously used in Fig. 4 at $t=70$ ns. Based on these fits, the motion of the plasma emissive surface over $45 \mathrm{~ns}$ is estimated at $0.24 \mathrm{~mm}$, corresponding to an average velocity of $0.53 \mathrm{~cm} / \mu \mathrm{s}$. This value is consistent with the one obtained by Coleman and co-coworkers who found an estimated plasma velocity of $0.59 \mathrm{~cm} / \mu \mathrm{s}$ at $3.8 \mathrm{MV}$ for a 1800 A electron diode [16]. Krasik and co-workers [27] inferred the plasma expansion velocity at $1.0 \pm 0.2 \mathrm{~cm} / \mu \mathrm{s}$ by monitoring the appearance of the $\mathrm{H} \alpha$ spectral line at different distances from the cathode.

\section{Characterizing the beam dynamics within the injector}

After the beam has been produced with the desired $2.6 \mathrm{kA}$ intensity, driven by the dose requirement for a given end-point energy, the beam must be injected through the accelerating section of the LIA in order to be accelerated and transported toward the $\mathrm{x}$-ray conversion target located $50 \mathrm{~m}$ downstream. The quality of the beam at the end of the injector (BPM 3, see Fig. 1) is in fact very critical for the transport properties and final beam focusing. The magnetic field induced by the extraction coil must allow optimal beam injection. Therefore the coil must be operated at an adequate driving current. The latter is determined using the time-dependent computational model developed in this study. The beam dynamics are simulated for various magnetic coil driving currents. Figure 7(a) shows the temporal profiles of the beam current for three different driving currents. In this figure, the beam current at BPM 1 , corresponding to the current upstream of the coil, is displayed along with the beam currents obtained downstream of the coil, for three different driving currents.

Figure 7(a) suggests that the beam current on the flattop is strongly dependent of the extraction solenoid driving current. If the latter is too low or too high, significant losses occur during transport from the diode to the end of the injector. Despite being based on hypotheses that are not fully valid within the diode, the envelope equation is quite useful for understanding and predicting the dynamics of the beam envelope downstream of the diode, beyond $\sim 17.2 \mathrm{~cm}$ [30-31]. The envelope equation is expressed as follows:

$$
\begin{aligned}
R^{\prime \prime}= & -\left[\frac{\gamma^{\prime}}{\beta^{2} \gamma}\right] R^{\prime}-\left[\frac{\gamma^{\prime \prime}}{2 \beta^{2} \gamma}\right] R-\left[\frac{e B z(0, z)}{2 \beta \gamma m_{0} c}\right]^{2} R \\
& +\frac{\varepsilon^{2}}{R^{3}}+\left[\frac{e \psi_{0}}{2 \pi \beta \gamma m_{0} c}\right]^{2} \frac{1}{R^{3}}+\frac{K}{R},
\end{aligned}
$$

where $R$ is the beam envelope radius and the prime symbol denotes a derivative taken with respect to $z, \beta=\mathrm{v} / \mathrm{c}$, $\gamma=1 /\left(1-\beta^{2}\right)^{1 / 2}$, and $K$ is the generalized perveance defined as

$$
K=\frac{e I}{2 \pi \varepsilon_{0} m_{0}(\beta \gamma c)^{3}} .
$$

Using the energy, emittance, currents and magnetic field values obtained from PIC simulations at $Z=17.2 \mathrm{~cm}$, the envelope equation is solved. Figures 7(b), 7(c), and 7(d) confirm the dependence of the transported beam current on the envelope equation and on the solenoid current. In this study, the dominants terms in Eq. (2) are the 4th, 5th and 7 th terms.

If the solenoid current is too low the beam space charge [7th term in Eq. (2)] exceeds the focusing strength [4th term in Eq. (2) and the beam blows up as confirmed by PIC calculations in Fig. 7(b). If the solenoid current is too high, the beam space charge becomes negligible compared to the focusing strength, resulting in beam overfocusing as illustrated in Fig. 7(c).

Furthermore, Fig. 7(e), which represents a snapshot of electron particle plot obtained from PIC LSP simulation at 201 A, 70 ns [Fig. 7(d)] along with the beam envelope curve obtained by solving the envelope equation, shows that the envelope code provides a reasonable description of the beam dynamics. Figure 7(e) shows that optimal transport is achieved with a $201 \mathrm{~A}$ driving current, since no loss occurs over the plateau.

It appears that the beam pulse is shortened between BPM 1 and BPM 3 for all driving currents. This result confirms the influence of energy on the beam envelope dynamics. Electrons produced during the rise and fall of the pulse are mostly lost in the pipe, inducing bremsstrahlung as shown by Coleman and co-workers [32]. In order to complement their study, simulations were carried out to extract all particles striking the tube, as can be seen from the time-integrated energy distribution of the "lost" electrons at 201 A shown in Fig. 8.

The "lost" electrons have energies below $3300 \mathrm{keV}$. The energy dissipated within the beam pipe due to electronic losses induces a temperature rise $\Delta T$. The latter 
(a)

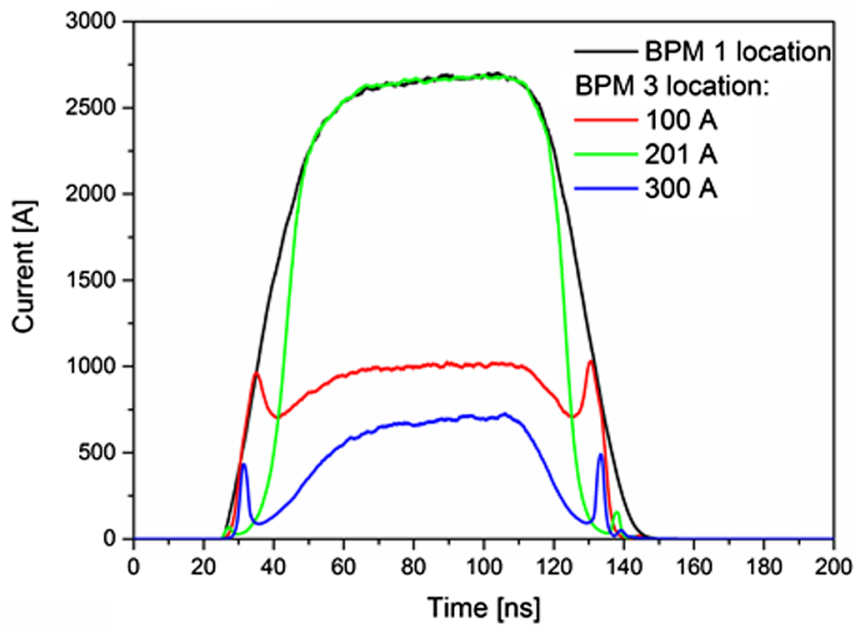

(b)

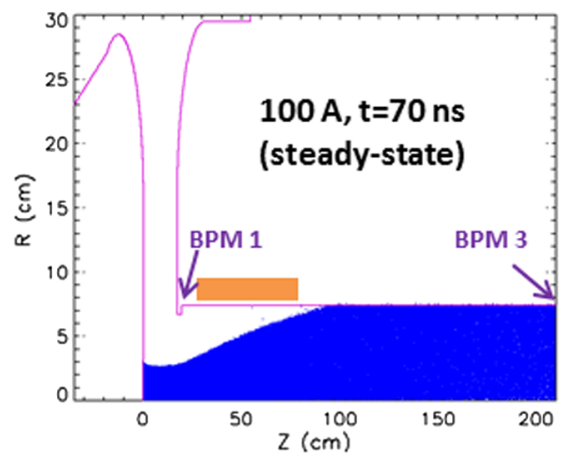

(e)

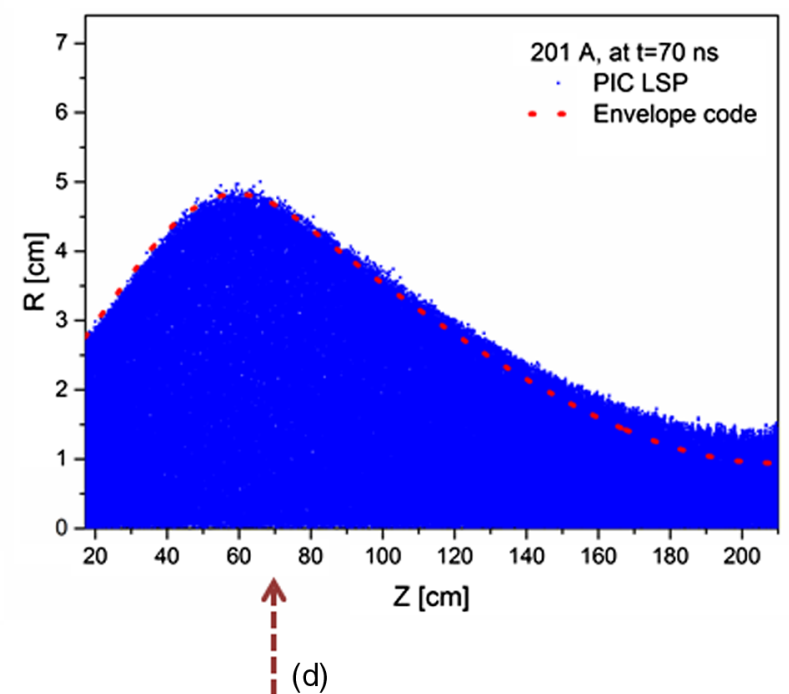

(d)
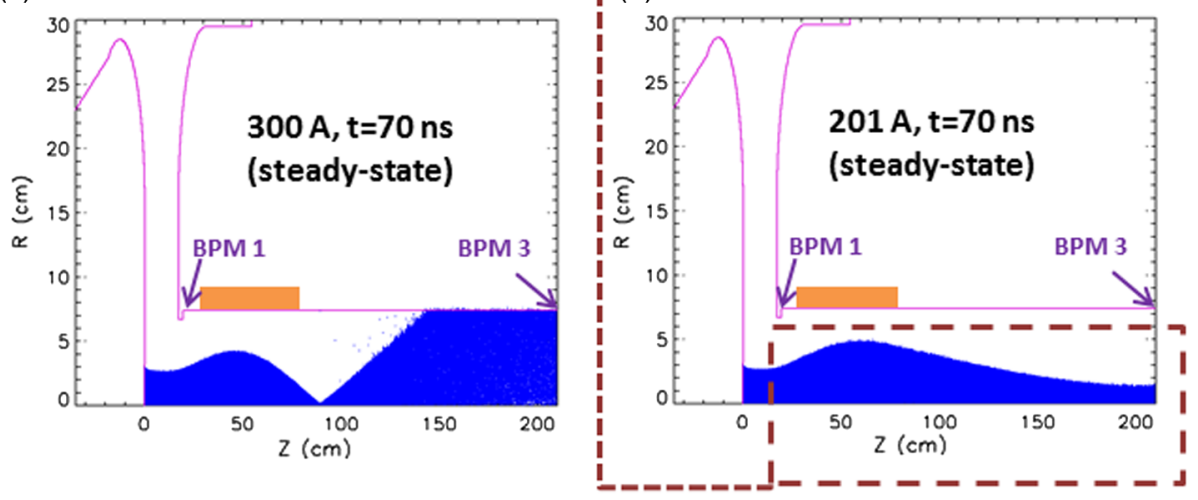

FIG. 7. (a) Simulated beam current at the BPM 3 location as a function of time for three different magnet driving currents: 100 A in red, $201 \mathrm{~A}$ in green and $300 \mathrm{~A}$ in blue. Beam current (in black) at the BPM 1 location after minor time rescaling for clarity (measurements were taken at different locations). Electron particle plots at $70 \mathrm{~ns}$ for $100 \mathrm{~A}$ (b), $300 \mathrm{~A}$ (c) and $201 \mathrm{~A}$ (d) driving currents. (e) Snapshot of electron particle plot obtained from PIC LSP simulation (in blue) at $201 \mathrm{~A}, 70 \mathrm{~ns}$, with the superimposed beam envelope curve (in red) obtained by solving the envelope equation.

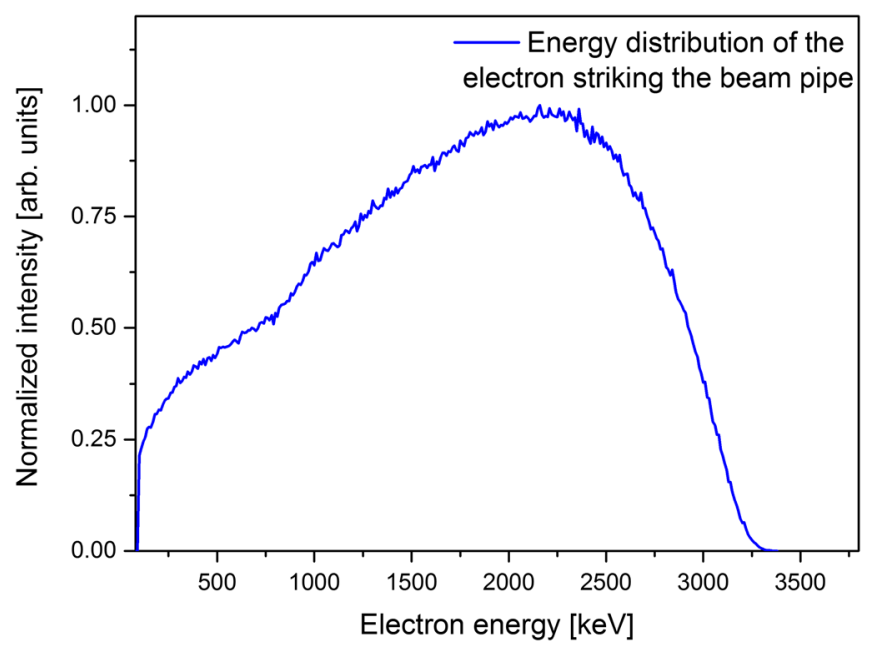

FIG. 8. Simulated time integrated energy distribution of the loss electrons striking the pipe. is calculated from the deposited energy per unit depth $(d E / d x)$ by each impacting electron, based on the formula

$$
\Delta T=\frac{d E}{d x} * \frac{e N_{e}}{\rho C_{p} \cos \theta},
$$

where $e$ is the electron charge, $N_{e}$ is the area density of electrons striking the pipe, $\rho$ is the material density, $\theta$ is the electron incidence angle and $C_{p}$ is the material specific heat. Simulation shows that the temperature rise resulting from the electron energy deposition is negligible $(<2 \mathrm{~K})$. Therefore, particle thermal desorption is negligible and does not affect the beam dynamics. The energy distribution in Fig. 8 confirms that all electrons produced over the duration of the plateau, with energy of about $3800 \mathrm{keV}$, are injected into the accelerator. Energy stability during the pulse is important for minimizing the $\mathrm{X}$-ray spot size. In Fig. 9, the injected charge at BPM 3, between 50 and $110 \mathrm{~ns}$ corresponding to the voltage plateau, is plotted for various 


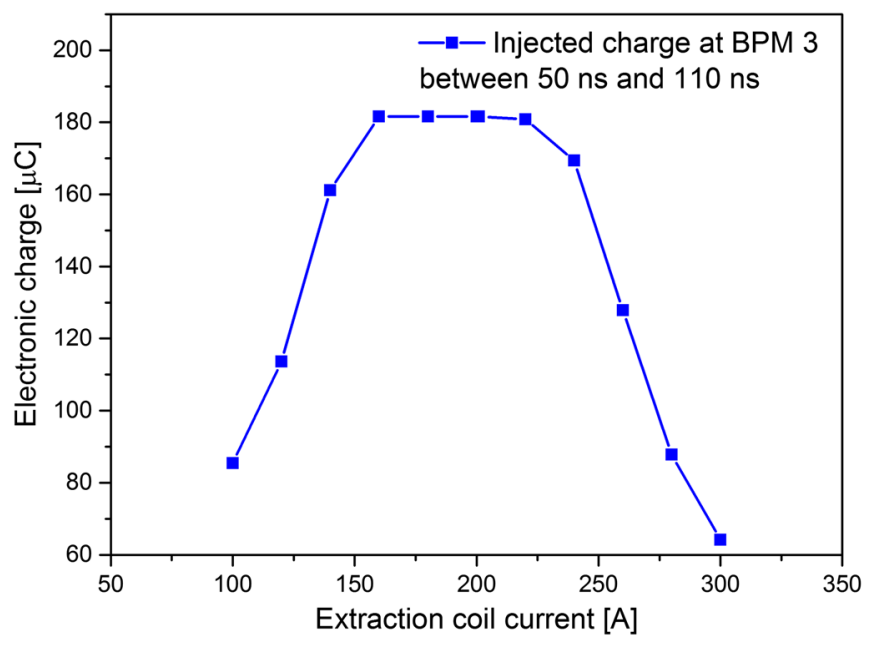

FIG. 9. Simulated electronic charge at BPM 3 integrated over 50 and 110 ns corresponding to the quasiflat part of the voltage pulse.

solenoid extraction currents. The injected charge is constant near $181.6 \mu \mathrm{C}$ suggesting that the beam injection efficiency is better achieved for driving currents ranging from 160 and $220 \mathrm{~A}$.

For the EPURE axis-1, the root-mean-square (rms) radius of the beam for different extraction coil currents at BPM 3 is deduced from the measured optical transition radiation (OTR) profile obtained with a $100 \mu \mathrm{m}$ thick aluminized kapton foil target. In Fig. 10, measurements are compared to a PIC simulation model which incorporates the OTR target.

As can be seen in Fig. 10, simulated rms beam radii are consistently smaller than the experimental values, even though they have similar profiles: the rms beam radius

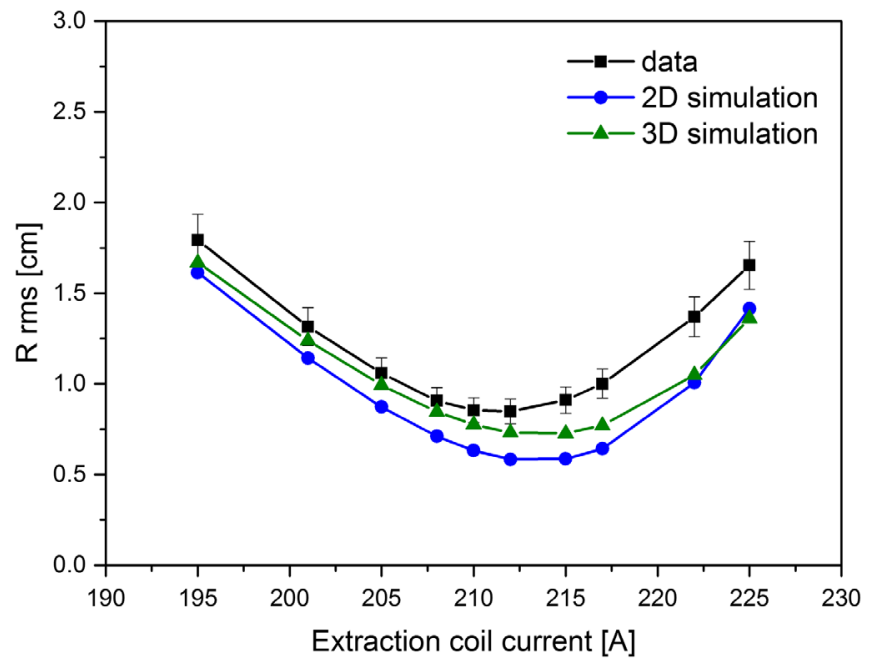

FIG. 10. Comparison of rms beam radii at BPM 3 for various extraction coil currents: measured (black squares) and simulated using an axisymmetric 2D geometry (blue circles) and a Cartesian 3D geometry (green triangles). decreases with increasing extraction coil currents up to a minimum. Then the rms radius increases beyond this minimum due to envelope dynamics.

The $3.8 \mathrm{MeV}, 2.6 \mathrm{kA}$ beam is likely to induce OTR target heating and gas desorption, producing ion backstreaming into the electron beam and increasing the rms size. Based on the 2D simulation, a maximal temperature rise of only $100 \mathrm{~K}$ was obtained for the OTR target for an extraction coil current of $212 \mathrm{~A}$, corresponding to the minimum rms radius. The discrepancy between simulated and experimental rms radii is consistently observed whether particles desorption occurs or not. This suggests that other mechanisms such as emittance growth during transport may, in addition to the beam target effects, contribute significantly to the observed discrepancy. In an attempt to investigate this aspect, we carried out 3D Cartesian simulation. As can be seen in Fig. 10, the 3D curve is closer to the experimental one. However, both simulations fail to properly reproduce the rms beam radii, especially beyond its minimum value. Although a higher electron temperature at the emission may partially explain the lower rms values obtained from simulations, it is very likely that the uniform emission model used in the simulation does not sufficiently account for all effects influencing the emittance growth, and hence tends to underestimate the emittance.

The "three gradients method" [33] is used in order to estimate the experimental emittance. The measured rms beam radius versus extraction coil current in Fig. 10 is fitted to a curve calculated from an envelope equation which depends on normalized root mean square (rms) emittance, initial rms beam radius and divergence at the anode. The same method is applied to the simulated rms radii curves in order to compare the experimental and simulated emittance values. From the experimental curve, a normalized emittance of $0.103 \mathrm{~cm}$ rad is inferred, while normalized emittances of $0.055 \mathrm{~cm} \mathrm{rad}$ and $0.080 \mathrm{~cm}$ rad are inferred from the axisymmetric 2D and 3D Cartesian simulations, respectively. In the absence of beam-target interaction effects [34] on the x-ray converter, the beam emittance is the ultimate limitation on $\mathrm{x}$-ray spot size.

\section{CONCLUSION}

Based on the particle-in-cell method, we developed a time-dependent simulation model of the electron beam dynamics within the injector. This model was used to design a new cathode with the objective to significantly increase the EPURE axis-1 beam intensity. Based on this model, a $3.175 \mathrm{~cm}$ radius cathode was designed, manufactured and tested on the EPURE axis-1 injector. This resulted in a significant increase of the beam intensity to $2.6 \mathrm{kA}$. The study shows that the beam intensity is strongly reduced when the recess increases, due to changes induced in the beam profile at the cathode. A very good agreement was obtained between simulated and measured currents at the exit of the extraction coil. The time-dependent 
computational model developed in this study enabled an estimate of the plasma velocity to $0.53 \mathrm{~cm} / \mu \mathrm{s}$. Utilizing the "three gradients" method for both the experimental and simulated root mean square radii allowed us to compare the experimental beam emittance $(0.103 \mathrm{~cm} \mathrm{rad})$ to values obtained through $2 \mathrm{D}$ axisymmetric $(0.055 \mathrm{~cm} \mathrm{rad})$ and $3 \mathrm{D}$ Cartesian simulations $(0.080 \mathrm{~cm}$ rad). Despite underestimating the beam emittance, the simulation is satisfactory for predicting the beam dynamics and properties, and especially the beam current within high current, high energy injectors. Efforts are under way for improving the 3D model by introducing a nonuniform emission model in the simulation and taking into account solenoid misalignments which may contribute to further emittance growth.

\section{ACKNOWLEDGMENTS}

All of the EPURE team is thanked for assistance with the experiments. Thierry Maillard is thanked for his continued support in simulation.

[1] C. Ekdahl, Modern electron accelerators for radiography, IEEE Trans. Plasma Sci. 30, 254 (2002).

[2] J. E. Coleman, D. C. Moir, C. A. Ekdahl, J. B. Johnson, B. T. McCuistian, G. W. Sullivan, and M. T. Crawford, Increasing the intensity of an induction accelerator and reduction of the beam breakup instability, Phys. Rev. ST Accel. Beams 17, 030101 (2014).

[3] C. Ekdahl, E. O. Abeyta, R. Archuleta, H. Bender, W. Broste, C. Carlson, G. Cook, D. Frayer, J. Harrison, T. Hughes, J. Johnson, E. Jacquez, B. Trent McCuistian, N. Montoya, S. Nath, K. Nielsen, C. Rose, M. Schulze, H. V. Smith, C. Thoma, and C. Y. Tom, Suppressing beamcentroid motion in a long-pulse linear induction accelerator, Phys. Rev. ST Accel. Beams 14, 120401 (2011).

[4] R. D. Scarpetti, J. K. Boyd, G. G. Earley, K. L. Griffin, R. G. Kerr, R. Kihara, M. M. Ong, J. M. Zentler, and N. L. Back, Upgrades to the LLNL flash X-ray induction accelerator (FXR), in Proceedings of the 11th IEEE International Pulsed Power Conference (1997), pp. 597-602.

[5] J. Deng, B. N. Ding, J. S. Shi, Y. He, J. Li, Q. Li, G. G. Cao, L. Wen, and G.S. Dai, Upgrading of linear induction accelerator x-ray facility (LIAXF), in Proceedings of the 19th International Linear Accelerators Conference, Chicago, IL, 1998 (NTIS, Springfield, VA, 1998), pp. 389390.

[6] M. Mouillet, R. Boivinet, F. Bombardier, J. Delvaux, E. Merle, J. C. Ribes, P. Anthouard, J. Bardy, A. Devin, and J. de Mascureau, First results of the AIRIX induction accelerator, in Proceedings of the 20th International Linac Conference, LINAC-2000, Monterey, CA, 2000 (SLAC, Menlo Park, CA, 2000), pp. 491-493.

[7] A. Georges, J. Beaubernard, V. Bernigaud, L. Buche, Y. Collet, S. Combacon, G. Grandpierre, B. Gouin, J. Kranzmann, J. Magnan, L. Magnin, D. Pierre, F. Poulet, R. Nicolas, Y. Tailleur, and J. L. Verstraete, Status on
AIRIX restart, in Proceedings of the XXVII linac conference, Geneva, 2014, pp. 462-464.

[8] G. A. Mesyats and D. I. Proskurovsky, Pulsed Electrical Discharge on Vacuum (Springer, Berlin, 1989).

[9] R. B. Miller, Mechanism of explosive electron emission for dielectric fiber (velvet) cathodes, J. Appl. Phys. 84, 3880 (1998).

[10] C. D. Child, Discharge from hot CaO, Phys. Rev. 32, 492 (1911).

[11] I. Langmuir, The effect of space charge and residual gases on thermionic currents in high vacuum, Phys. Rev. 2, 450 (1913).

[12] H. R. Jory and A. W. Trivelpiece, Exact relativistic solution for the one-dimensional diode, J. Appl. Phys. 40, 3924 (1969).

[13] Y. Y. Lau, D. Chemin, D. G. Colombant, and P. T. Ho, Quantum Extension of Child-Langmuir Law, Phys. Rev. Lett. 66, 1446 (1991).

[14] Y. Y. Lau, Simple Theory for the Two-Dimensional ChildLangmuir Law, Phys. Rev. Lett. 87, 278301 (2001).

[15] J. W. Luginsland, Y. Y. Lau, and R. M. Gilgenbach, TwoDimensional Child-Langmuir Law, Phys. Rev. Lett. 77, 4668 (1996).

[16] J. E. Coleman, D. C. Moir, M. T. Crawford, D. R. Welch, and D. T. Offermann, Temporal response of a surface flashover on a velvet cathode in a relativistic diode, Phys. Plasmas 22, 033508 (2015).

[17] T. Houck, C. Brown, D. Fleming, B. Kreitzer, K. Lewis, M. Ong, and J. Zentler, Design of a high field stress, velvet cathode for the flash $\mathrm{x}$-ray (FXR) induction accelerator, in Proceedings of the 22nd Particle Accelerator Conference, PAC-2007, Albuquerque, NM (IEEE, New York, 2007).

[18] N. Pichoff, M. Caron, A. Compant-La-Fontaine, D. Paradis, L. Hourdin, F. Cartier, D. Collignon, G. Grandpierre, and M. Mouillet, Contributors to AIRIX focal spot size, in Proceedings of the 10th European Particle Accelerator Conference, Edinburgh, Scotland, 2006 (EPS-AG, Edinburgh, Scotland, 2006), pp. 2164-2166.

[19] LSP is a software product of Orbital ATK.

[20] J. M. Plewa, O. Eichwald, M. Yousfi, G. Wattieaux, S. Cartier, F. Cartier, F. Poulet, V. Bernigaud, M. Ribière, R. Delaunay, T. D'Almeida, and R. Maisonny, Modeling and experimental characterization of the plasma produced by a velvet cathode in a linear induction accelerator (to be published).

[21] J. A. Halbleib, R. P. Kensek, T. A. Mehlhorn, G. D. Valdez, S. M. Seltzer, and M. J. Berger, ITS: The integrated TIGER series of electron/photon transport codes-Version 3.0, IEEE Trans. Nucl. Sci. 39, 1025 (1992).

[22] M. Reiser, Theory and Design of Charged Particle Beams, 2nd ed. (Wiley, New York, 2008).

[23] S. M. Lund, Nonlinear optics of solenoid magnets in Proceedings of the 6th IPAC Conference, Richmond, VA, 2015.

[24] T. P. Hugues, R. L. Carlson, and D. C. Moir, Highbrightness electron-beam generation and transport, J. Appl. Phys. 68, 2562 (1990).

[25] F. Hegeler, M. Friedman, M. C. Myers, J. D. Sethian, and S. B. Swanekamp, Reduction of enhanced edge emission in a large area electron beam, Phys. Plasmas 9, 4309 (2002). 
[26] M. Caron, F. Bombardier, E. Merle, Ch. Noël, O. Pierret, R. Rosol, C. Vermare, N. Pichoff, A. Piquemal, and D. C. Moir, Comparison of 2 cathode geometries for high current (2 kA) diodes, in Proceedings of LINAC 2004, Lübeck, Germany (2004), p. 654.

[27] Ya. E. Krasik, J. Z. Gleizer, D. Yarmolich, A. Krokhmal, V. Ts. Gurovich, S. Efimov, J. Felsteiner, V. Bernshtam, and Yu. M. Saveliev, Characterization of the plasma on dielectric fiber (velvet) cathodes, J. Appl. Phys. 98, 093308 (2005).

[28] D. A. Shiffler, J. W. Luginsland, R. J. Umstatt, M. LaCour, K. Golby, M. D. Haworth, M. Ruebush, D. Zagar, A. Gibbs, and T. A. Spencer, Effects of anode materials on the performance of explosive field emission diodes, IEEE Trans. Plasma Sci. 30, 1232 (2002).

[29] J. E. Coleman, D. C. Moir, C. A. Ekdahl, J. B. Johnson, B. T. McCuistian, and M. T. Crawford, Explosive emission and gap closure from a relativistic electron beam diode, in
Proceedings of the 19th PPC conference (IEEE, 2013), pp. 390-394.

[30] M. Reiser, Theory and Design of Charged Particle Beams, Second, Updated and Expanded Edition (John Wiley and Sons Inc., New York, 2008), p. 65.

[31] S. Humphries Jr., Charged Particle Beams (John Wiley and Sons Inc., New York, 2002), p. 401.

[32] J. E. Coleman, D. R. Welch, and C. L. Miller, Scattered hard $\mathrm{x}$-ray and $\gamma$-ray generation from a chromatic electron beam, J. Appl. Phys. 118, 184505 (2015).

[33] J. Bardy, C. Bonnafond, A. Devin, E. Merle, and D. Villate, Status of AIRIX alignment and high current electron beam diagnostics, in Proceedings of the 5th EPAC conference, 1996, p. 1588

[34] C. Vermare, H. A. Davis, D. C. Moir, and T. P. Hughes, Ion emission from solid surfaces induced by intense electron beam impact, Phys. Plasmas 10, 277 (2003). 